\title{
Sorafenib versus Hepatic Arterial Infusion Chemotherapy as Initial Treatment for Hepatocellular Carcinoma with Advanced Portal Vein Tumor Thrombosis
}

\author{
Michihisa Moriguchi ${ }^{a}$ b Takeshi Aramaki ${ }^{a}$ Hideyuki Nishiofuku ${ }^{c}$ \\ Rui Sato $^{a}$ Koiku Asakura ${ }^{d}$ Kanji Yamaguchi $^{b}$ Toshihiro Tanaka ${ }^{c}$ \\ Masahiro Endo ${ }^{d}$ Yoshito Itoh ${ }^{b}$ \\ ${ }^{a}$ Division of Interventional Radiology, Shizuoka Cancer Center, Shizuoka, ${ }^{b}$ Department of \\ Molecular Gastroenterology and Hepatology, Kyoto Prefectural University of Medicine \\ Graduate School of Medical Science, Kyoto, ' Department of Radiology, Nara Medical \\ University, Kashihara, and d Division of Diagnostic Radiology, Shizuoka Cancer Center, \\ Shizuoka, Japan
}

\section{Keywords}

Hepatic arterial infusion chemotherapy · Hepatocellular carcinoma - Low-dose 5-fluorouracil and cisplatin · Portal vein tumor thrombus · Sorafenib

\begin{abstract}
Objective: To investigate the validity of hepatic arterial infusion chemotherapy with low-dose 5-fluorouracil and cisplatin (LFP) versus sorafenib as first-line treatment for hepatocellular carcinoma (HCC) with portal vein tumor thrombosis (Vp3, Vp4). Patients and Methods: We retrospectively reviewed the cases of Child-Pugh A advanced HCC with Vp3 or Vp4 treated with LFP or sorafenib between October 2002 and December 2013. Results: There were 32 patients in the LFP group and 14 patients in the sorafenib group. The objective response rate/ disease control rate was $31.3 / 56.3 \%$ in the LFP group and $0 / 28.6 \%$ in the sorafenib group. The median survival time (MST) (309 vs. 120 days; $p=0.009$ ) and the median time to treatment failure (109 vs. 37 days; $p=0.022$ ) were significantly longer in the LFP group than in the sorafenib group. In the LFP group, a relatively favorable outcome (MST, 622 days) was obtained among the response cases. Among the nonresponse cases in the LFP group, at the time of cessation of LFP, $70.4 \%$ of cases were Child-Pugh A and $88.9 \%$ of cases maintained a score of $\leq 7$ points; of the cases in whom Child-Pugh A was maintained, the survival period from the time of LFP discontinuation was significantly longer in the cases in whom sorafenib was introduced as a secondary treatment after LFP than in the cases treated with best supportive care
\end{abstract}


(220 vs. 89 days; $p=0.002$ ). The main adverse event with LFP was grade 3 or higher cytopenia, which was manageable, and adverse event-induced discontinuation was significantly lower as compared with sorafenib ( $p=0.002)$. Conclusion: For the treatment of HCC with Vp3/Vp4, it is desirable to initially use LFP and then immediately change to sorafenib if no response is obtained.

(C) 2017 S. Karger AG, Basel

\section{Introduction}

Sorafenib is a multikinase inhibitor that slows tumor cell growth and suppresses neovascularization [1]. Two phase III trials have shown a survival benefit of sorafenib in advanced hepatocellular carcinoma (HCC) [2, 3]. In addition, a subanalysis of the Study of Heart and Renal Protection (SHARP) trial showed a survival benefit of sorafenib in HCC with macroscopic vascular invasion [4]. Currently, sorafenib is the standard treatment against ChildPugh A advanced HCC with portal vein tumor thrombosis.

In Japan, hepatic arterial infusion chemotherapy (HAIC) is frequently used against advanced HCC with portal vein tumor thrombosis, with a high response rate of 33-48\% and favorable survival periods in cases showing a response [5-7]. For this reason, the treatment is cited as an option in consensus-based guidelines [8]. Low-dose 5 -fluorouracil and cisplatin (LFP), administered after placement of a reservoir catheter system, is a relatively commonly used regimen [5-7]. While its benefits have not been confirmed in phase III trials, it is often used in anticipation of its tumor reduction effect, even since the advent of sorafenib.

The prognosis of HCC with macrovascular invasion, namely Vp3 (tumor thrombus involving the first branches of the portal vein) and Vp4 (tumor thrombus involving the main trunk of the portal vein), is extremely poor $[9,10]$, and the efficacy of sorafenib is limited [11, 12]. Therefore, defining an effective treatment is a high priority. In consensus-based guidelines in Japan, sorafenib and HAIC are the 2 treatment options for HCC with Vp3, while only HAIC is considered for HCC with Vp4. However, on a global scale, sorafenib is considered the only standard treatment for macroscopic vascular invasion [13]. Yet, clinically it is an extremely important decision whether to initially use sorafenib, which is the global standard, or HAIC, which has a high response rate.

In this study, we investigated the validity of initial treatment with LFP for Child-Pugh A advanced HCC with Vp3 or Vp4. This paper describes the results of a comparison between cases treated with LFP and cases treated with sorafenib.

\section{Patients and Methods}

We conducted a retrospective study of the results of cases using LFP as the initial treatment, compared with sorafenib cases, for Child-Pugh A advanced HCC with Vp3 or Vp4 during the period from October 2002 to December 2013. The inclusion criteria were as follows: (1) clinically diagnosed HCC with Vp3 or Vp4 as determined by blood testing (viral markers, tumor markers) and dynamic contrast-enhanced computed tomography or magnetic resonance imaging; (2) Child-Pugh A; (3) Eastern Cooperative Oncology Group (ECOG) performance status (PS) of 0 or 1 ; (4) white blood cell count $\geq 2,000 / \mu \mathrm{L}$, hemoglobin $\geq 8.0 \mathrm{~g} / \mathrm{dL}$, and platelet count $\geq 6.0 \times 10^{4} / \mu \mathrm{L}$; (4) inability to undergo surgical resection, or refractory to or unsuitable for transarterial chemoembolization; and (5) no concomitant use of other treatments. LFP was chosen before sorafenib became commercially available. After sorafenib had become available, both options (LFP and sorafenib) were explained to each patient, and treatment was decided based on the patient's choice. 


\section{Liver Cancer}

LFP Administration

LFP was administered following placement of a reservoir catheter system, up until the fourth week, in accordance with a previously reported regimen [7] consisting of 4 cycles of intra-arterial infusion of cisplatin (10 $\mathrm{mg}$ in $1 \mathrm{~h}$ ), followed by 5 -fluorouracil ( $250 \mathrm{mg}$ in $5 \mathrm{~h}$ ) on days 1 through 5 , and cessation on days 6 and 7. For the fifth week onwards, it was administered once a week on day 1 only. Treatment was continued until tumor progression occurred or adverse events impeding continuation developed.

\section{Sorafenib Administration}

Generally, administration was commenced at $800 \mathrm{mg} /$ day, with rest periods and dose reductions implemented in the case of adverse events. There were also cases in whom administration was commenced at $400 \mathrm{mg} /$ day, at the primary physician's discretion. Dose escalation was attempted whenever possible. Treatment was continued until tumor progression occurred or adverse events impeding continuation developed.

\section{Additional or Subsequent Therapy}

Additional or subsequent therapy was indicated for patients with a PS of 0-2 or Child-Pugh A or B, or those who maintained bone marrow function/renal function. However, the condition where Child-Pugh A was maintained was set to be required for introducing sorafenib following LFP. When the therapy was effective and an improvement (e.g., downstaging) was obtained, surgical resection, radiofrequency ablation, or transarterial chemoembolization was performed. On the other hand, when the therapy was not effective, HAIC (a single shot of cisplatin, continuous infusion of 5-fluorouracil, continuous infusion of 5-fluorouracil with subcutaneous interferon injection), oral administration of tegafur and uracil, or radiation therapy was performed based on the patient's choice.

\section{Evaluation and Statistical Analysis}

Therapeutic efficacy was assessed with contrast-enhanced computed tomography every 4-6 weeks. Response Evaluation Criteria in Solid Tumors (RECIST) v1.0 was used for an assessment of direct antitumor effects, and the objective response rate and the disease control rate were calculated. The Kaplan-Meier method was used to analyze overall survival (OS), median survival time (MST), and time to treatment failure (TTF), and the log-rank test was used to compare between the LFP group and the sorafenib group. TTF was defined as the period up until treatment discontinuation due to tumor progression, or discontinuation due to adverse events. The factors contributing to survival were identified using univariate and multivariate analyses. $p$ values $<0.05$ were considered to be statistically significant. Factors with $p<0.10$ in the univariate analysis were considered for entry into the multivariate analysis. We looked at the Child-Pugh score at the time of discontinuation of initial treatment and determined changes as compared to before treatment as well as secondary treatments. The Kaplan-Meier method was also used to analyze survival times for those cases in whom Child-Pugh A had been maintained at the time of discontinuation in the LFP group and sorafenib was chosen as the secondary treatment, and those cases in whom best supportive care (BSC) was chosen. SPSS v19 was used for all analyses.

This study was conducted in accordance with the Declaration of Helsinki and was approved by the Ethics Committee of Shizuoka Cancer Center.

\section{Results}

The patient characteristics are shown in Table 1. There were 32 patients in the LFP group and 14 patients in the sorafenib group. The initial dose of sorafenib was $400 \mathrm{mg}$ in 7 patients and $800 \mathrm{mg}$ in another 7 patients. The median age was 65 (40-81) years in the LFP group and 68 (53-82) years in the sorafenib group. Most patients were men (male/female ratio, 29/3 in the LFP group and 12/2 in the sorafenib group). The number of patients with a PS of $0 / 1$ was 19/13 in the LFP group and 6/8 in the sorafenib group. There were significantly more Vp4 patients in the sorafenib group than in the LFP group (Vp3/Vp4 ratio, 25/7 in the LFP group vs. 5/9 in the sorafenib group; $p=0.008$ ). There was no significant difference in extrahepatic spread (EHS) between the 2 groups (EHS+/EHS- ratio, 7/25 in the LFP group vs. 5/9 
Moriguchi et al.: Sorafenib versus Hepatic Arterial Infusion Chemotherapy as Initial

Table 1. Overall patient characteristics

\begin{tabular}{llll}
\hline & LFP $(n=32)$ & Sorafenib $(n=14)$ & $p$ value \\
\hline Age, years & $65(40-81)$ & $68(53-82)$ & 0.293 \\
Sex, male/female & $29 / 3$ & $12 / 2$ & 0.633 \\
PS 0/1 & $19 / 13$ & $6 / 8$ & 0.748 \\
Vp3/Vp4 & $25 / 7$ & $5 / 9$ & 0.008 \\
EHS+/EHS- & $7 / 25$ & $5 / 9$ & 0.567 \\
Main tumor size, mm & $74.7(0-179.1)$ & $65.8(32.7-108.0)$ & 0.567 \\
Tumor burden, $\leq 50 \% />50 \%$ & $15 / 17$ & $6 / 8$ & 0.801 \\
Child-Pugh score 5/6 & $11 / 21$ & $4 / 10$ & 1.000 \\
Bilirubin, mg/dL & $0.7(0.3-1.4)$ & $0.8(0.4-1.4)$ & 0.781 \\
Albumin, g/dL & $3.6(2.9-4.7)$ & $3.6(3.3-4.2)$ & 0.706 \\
Prothrombin time, $\%$ & $82(51-99)$ & $87(51-122)$ & 0.142 \\
White blood cells, / $\mu \mathrm{L}$ & $4,900(1,650-10,590)$ & $5,260(2,590-7,050)$ & 0.830 \\
Hemoglobin, g/dL & $12.5(8.1-15.2)$ & $12.3(8.8-15.5)$ & 0.474 \\
Platelets, / $\mu \mathrm{L}$ & $15.7(7.3-45.9)$ & $12.4(10.6-24.3)$ & 0.551 \\
Etiology, HBV/HCV/NBNC & $12 / 7 / 13$ & $4 / 8 / 2$ & 0.0495 \\
Alpha-fetoprotein, ng/mL & $466.1(5.1-340,140)$ & $416.9(4.3-211,634)$ & 0.445 \\
PIVKA-II, mAU/mL & $2,570(23-307,000)$ & $18,800(414-631,000)$ & 0.052 \\
History of TACE, $+/-$ & $12 / 20$ & $9 / 5$ & 0.117 \\
Initial dose, $400 \mathrm{mg} / 800 \mathrm{mg}$ & - & $7 / 7$ & - \\
\hline
\end{tabular}

The numerical data represent the numbers of cases or the median values. Tumor burden means the relative rate of tumor occupying the liver. EHS, extrahepatic spread; HBV, hepatitis B virus; HCV, hepatitis C virus; LFP, low-dose 5-fluorouracil and cisplatin; NBNC, non-B non-C; PIVKA-II, protein induced by vitamin $\mathrm{K}$ absence or antagonist II; PS, performance status; TACE, transarterial chemoembolization; Vp3, tumor thrombus involving the first branches of the portal vein; $\mathrm{Vp} 4$, tumor thrombus involving the main trunk of the portal vein.

in the sorafenib group; $p=0.567$ ). There were no differences in the main tumor size or tumor burden (the relative rate of tumor occupying the liver) between the groups (main tumor size, $p=0.567$; tumor burden, $p=0.801$ ). In addition, there was a significant difference in viral markers between the 2 groups (hepatitis B virus/hepatitis C virus/non-B non-C, 12/7/13 in the LFP group and 4/8/2 in the sorafenib group; $p=0.0495$ ).

The number of complete response (CR), partial response (PR), stable disease (SD), progressive disease (PD), and not evaluated cases was 1, 9, 8, 12, and 2 in the LFP group, and $0,0,4,7$, and 3 in the sorafenib group. The objective response rate and the disease control rate were 31.3 and $56.3 \%$ in the LFP group, and 0 and $28.6 \%$ in the sorafenib group. The analysis of the relationship between direct antitumor effects and main tumor size showed that in the LFP group, the cases in whom the main tumor size was relatively small tended to respond to therapy (CR, PR/SD, PD [main tumor size $\leq 74.7$ vs. $>74.7 \mathrm{~mm}$ ]; $8 / 2$ vs. $2 / 20 ; p=$ 0.040 ), whereas there was no relationship between direct antitumor effects and tumor burden (CR, PR/SD, PD [tumor burden $\leq 50$ vs. $>50 \%$ ]; $7 / 10$ vs. $3 / 12 ; p=0.197$ ).

Figure 1 shows the OS (Fig. 1a) and the TTF (Fig. 1b) for the LFP and sorafenib groups. The MST ( 309 vs. 120 days; $p=0.009$ ) and the median TTF (109 vs. 37 days; $p=0.022$ ) were significantly longer in the LFP group than in the sorafenib group. In the LFP group, a relatively favorable outcome (MST, 622 days) was obtained in the response cases (Fig. 1c).

Figure 2 shows the OS for the LFP and sorafenib groups according to Vp3/Vp4 and EHS+/ EHS-. While there was no significant difference between Vp3 and Vp4, the LFP group tended to show longer survival times (MST of Vp3, 309 and 221 days for the LFP and sorafenib groups, respectively, $p=0.384$; MST of Vp4, 412 and 94 days for the LFP and sorafenib groups, 
Moriguchi et al.: Sorafenib versus Hepatic Arterial Infusion Chemotherapy as Initial

Treatment for Hepatocellular Carcinoma with Advanced Portal Vein Tumor Thrombosis
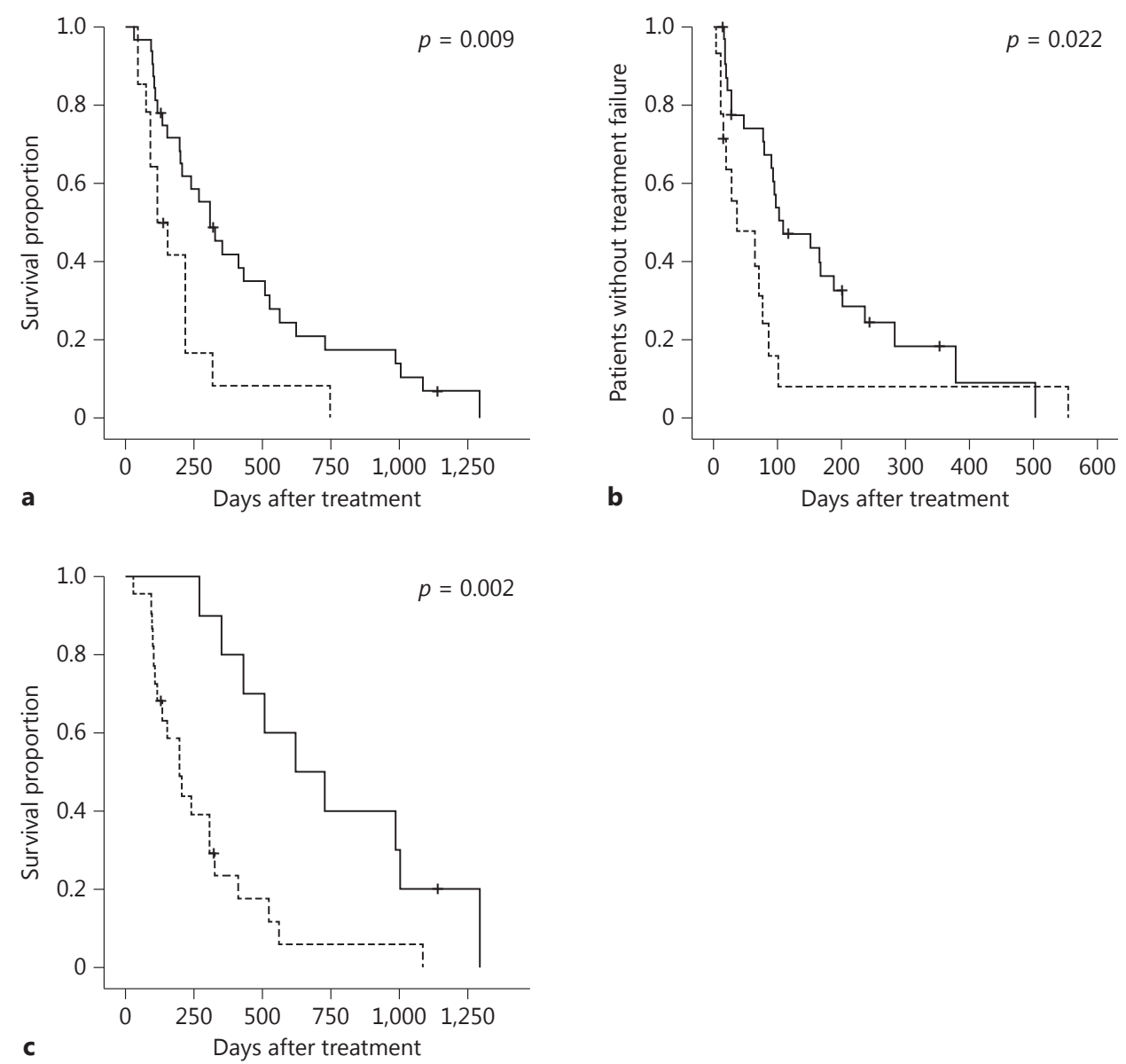

Fig. 1. a, b Kaplan-Meier plots of overall survival (a) and time to treatment failure (b) in the low-dose 5 -fluorouracil and cisplatin (LFP) $(n=32)$ and sorafenib $(n=14)$ groups. The LFP group is represented by a solid line and the sorafenib group by a dotted line. The median survival time (309 vs. 120 days; $p=0.009$ ) and the median time to treatment failure (109 vs. 37 days; $p=0.022$ ) were significantly longer in the LFP group than in the sorafenib group. c Overall survival in the LFP response and nonresponse groups. The response group is represented by a solid line and the nonresponse group by a dotted line. In the LFP group, a relatively favorable outcome (median survival time, 622 days) was obtained in the response cases.

respectively, $p=0.057)$. In the EHS- group, the prognosis was significantly more favorable in the LFP group (MST, 412 and 217 days for the LFP and sorafenib groups, respectively; $p=$ 0.037). However, in the EHS+ group, there was no difference between the LFP and sorafenib groups (MST, 117 and 120 days, respectively; $p=0.272$ ).

Figure 3 shows the survival curves for the LFP group, for cases in whom Child-Pugh A was maintained at the time of discontinuation due to PD or adverse events, in BSC cases or following introduction of secondary treatments such as sorafenib. The prognosis was significantly favorable in the sorafenib group as compared with the BSC group (MST, 220 and 89 days, respectively; $p=0.002$ ).

Univariate analysis revealed PS, Child-Pugh score, EHS status, main tumor size, protein induced by vitamin $\mathrm{K}$ absence or antagonist II level, and initial treatment as significant factors contributing to OS. Multivariate analysis revealed PS ( $p=0.017$, hazard ratio [HR] $2.660,95 \%$ 
Moriguchi et al.: Sorafenib versus Hepatic Arterial Infusion Chemotherapy as Initial

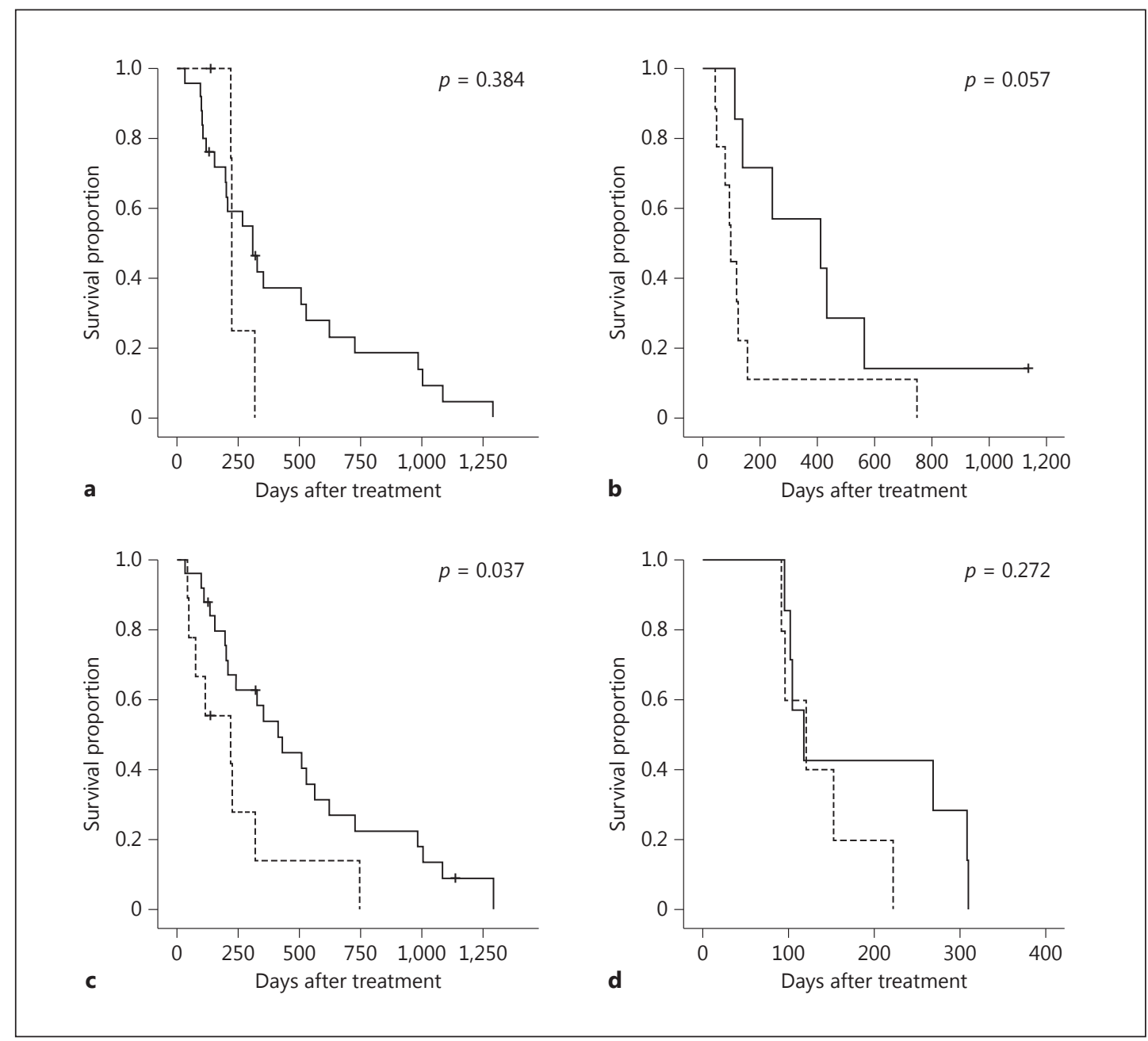

Fig. 2. Kaplan-Meier plot of overall survival according to Vp3/Vp4 and extrahepatic spread positivity (EHS+) and negativity (EHS-) in the low-dose 5-fluorouracil and cisplatin (LFP) and sorafenib groups. a Vp3 cases. b Vp4 cases. c EHS- cases. d EHS+ cases. The LFP groups are represented by a solid line and the sorafenib groups by a dotted line. $\mathbf{a}, \mathbf{b}$ The LFP group tended to show longer survival times (median survival time for Vp3, 309 and 221 days for the LFP and the sorafenib group, respectively, $p=0.384$; median survival time for Vp4, 412 and 94 days for the LFP and the sorafenib group, respectively, $p=0.057$ ). c The prognosis was significantly more favorable in the LFP group (median survival time, 412 and 217 days for the LFP and the sorafenib group, respectively; $p=0.037$ ). $\mathbf{d}$ There was no difference between the LFP and the sorafenib group (median survival time, 117 and 120 days, respectively; $p=0.272$ ).

confidence interval [CI] 1.188-5.957), Child-Pugh score ( $p<0.001$, HR 4.204, 95\% CI 1.9039.288), tumor burden ( $p=0.046$, HR 2.530,95\% CI 1.015-6.309), and initial treatment for HCC with Vp3/Vp4 ( $p=0.005$, HR 0.249, 95\% CI 0.095-0.665) as significant factors (Table 2).

In terms of grade 3 or higher adverse events, cytopenia was common in the LFP group, whereas in the sorafenib group, there were high numbers of hepatic function deterioration and systemic fatigue. In the sorafenib group, discontinuation of treatment due to a decline in PS was seen in 1 patient. Discontinuation due to adverse events was significantly more frequent in the sorafenib group than in the LFP group ( 7 vs. 3 cases; $p=0.002$ ) (Table 3).

Table 4 shows the Child-Pugh score in the LFP and sorafenib groups at the point of discontinuation due to adverse events or tumor progression. Patients whose Child-Pugh classifi- 


\section{Liver Cancer}

Fig. 3. Survival curves following discontinuation of low-dose 5fluorouracil and cisplatin (LFP). The sorafenib group is represented by a solid line and the best supportive care group by a dotted line. After discontinuation of LFP, the prognosis was significantly favorable in the group in which sorafenib was introduced as secondary treatment compared to the best supportive care group (median survival time, 220 and 89 days, respectively; $p=0.002$ ).

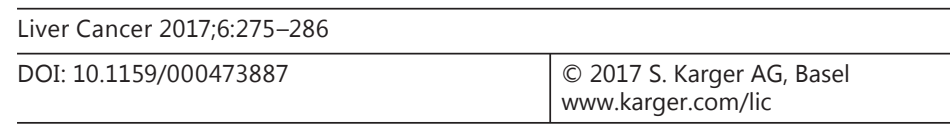

Moriguchi et al.: Sorafenib versus Hepatic Arterial Infusion Chemotherapy as Initial Treatment for Hepatocellular Carcinoma with Advanced Portal Vein Tumor Thrombosis

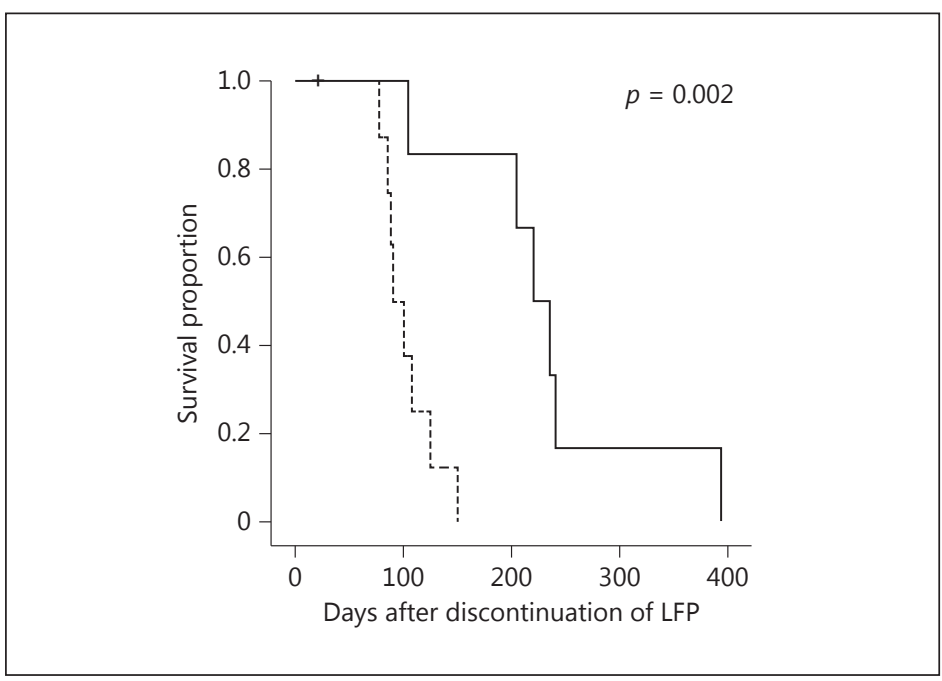

cation was degraded from A to B, and those who had worsening Child-Pugh scores of $\geq 2$ points were seen more commonly in the sorafenib group than in the LFP group $(p<0.001$ and $p=0.002$, respectively). Furthermore, secondary treatments were introduced more often in the LFP group, and sorafenib was the most commonly introduced treatment. In contrast, BSC was chosen in most cases in the sorafenib group.

\section{Discussion}

HCC with Vp3/Vp4 has a poor prognosis $[9,10]$, with expected outcomes being rapid deterioration of hepatic function and an associated decline in PS, and it is not uncommon for treatment opportunities to be missed. Even with the introduction of treatment, if there is no effect and the disease condition deteriorates, it is expected that the introduction of secondary treatments will be extremely difficult.

Globally, sorafenib is the standard treatment for HCC with Vp3/Vp4, but its effect is limited $[11,12]$, and from the perspective of adverse events, there is always the possibility that its full potential will not be realized. In our study, sorafenib treatment was associated with many cases of discontinuation due to adverse events and short TTF periods. With an MST of 4 months, our results cannot be said to be favorable. When sorafenib was ceased, the introduction of secondary treatments was difficult due to a decline in hepatic functional reserve, fatigue, and a decline in PS, as in previous reports [14]. In HCC with Vp3/Vp4, if the thrombi cannot be reduced, portal vein blood flow will decrease and hepatic function will be reduced further. Therefore, a treatment method with a strong tumor reduction effect is considered desirable for HCC with Vp3/Vp4.

The results with LFP in our study were a response rate of $31.3 \%$ and an MST of 10.3 months, which are similar to those in previous reports [5-7]. In response cases, relatively favorable prognoses were obtained [7]. With regard to cases in whom a response was not obtained, once sorafenib was introduced as a secondary treatment, and a more favorable prognosis was obtained as compared to the nontreatment group. At the time of discontinuation due to tumor progression or adverse events, in approximately $90 \%$ of cases, the ChildPugh score was maintained at $\leq 7$ points, and it was thought that overall, sorafenib introduction secondary to LFP treatment was possible [15]. In the present study, for secondary treatments following LFP, regardless of the maintenance of hepatic functional reserve, BSC 
Table 2. Factors contributing to overall survival by univariate and multivariate analysis
Moriguchi et al.: Sorafenib versus Hepatic Arterial Infusion Chemotherapy as Initial Treatment for Hepatocellular Carcinoma with Advanced Portal Vein Tumor Thrombosis

\begin{tabular}{|c|c|c|c|c|}
\hline & MST & $p$ value & HR & $95 \% \mathrm{CI}$ \\
\hline \multicolumn{5}{|l|}{ Univariate analysis } \\
\hline Initial treatment & & 0.009 & 2.427 & $1.217-4.838$ \\
\hline Sorafenib & 120 & & & \\
\hline LFP & 309 & & & \\
\hline Performance status & & 0.007 & 0.426 & $0.224-0.809$ \\
\hline 0 & 412 & & & \\
\hline 1 & 198 & & & \\
\hline Age & & 0.751 & 0.904 & $0.483-1.691$ \\
\hline$<70$ years & 268 & & & \\
\hline$\geq 70$ years & 217 & & & \\
\hline Sex & & 0.072 & 2.923 & $0.865-9.870$ \\
\hline Male & 221 & & & \\
\hline Female & 224 & & & \\
\hline Child-Pugh score & & 0.001 & 0.311 & $0.154-0.631$ \\
\hline 5 & 430 & & & \\
\hline 6 & 152 & & & \\
\hline Vp & & 0.270 & 0.700 & $0.370-1.325$ \\
\hline 3 & 308 & & & \\
\hline 4 & 120 & & & \\
\hline Extrahepatic spread & & 0.001 & 0.292 & $0.135-0.633$ \\
\hline Negative & 326 & & & \\
\hline Positive & 117 & & & \\
\hline Main tumor size & & 0.014 & 0.441 & $0.225-0.864$ \\
\hline$\leq 66.5 \mathrm{~mm}$ & 430 & & & \\
\hline$>66.5 \mathrm{~mm}$ & 206 & & & \\
\hline Tumor burden & & 0.059 & 0.542 & $0.284-1.033$ \\
\hline$\leq 50 \%$ & 309 & & & \\
\hline$>50 \%$ & 200 & & & \\
\hline TACE history & & 0.448 & 1.275 & $0.679-2.394$ \\
\hline Negative & 240 & & & \\
\hline Positive & 221 & & & \\
\hline Hepatitis B virus & & 0.515 & 0.806 & $0.420-1.546$ \\
\hline Positive & 240 & & & \\
\hline Negative & 217 & & & \\
\hline Hepatitis C virus & & 0.905 & 0.961 & $0.496-1.860$ \\
\hline Positive & 221 & & & \\
\hline Negative & 240 & & & \\
\hline Non-B non-C & & 0.460 & 0.778 & $0.400-1.517$ \\
\hline Positive & 206 & & & \\
\hline Negative & 224 & & & \\
\hline Alpha-fetoprotein & & 0.617 & 0.855 & $0.461-1.585$ \\
\hline$\leq 466.1 \mathrm{ng} / \mathrm{mL}$ & 318 & & & \\
\hline$>466.1 \mathrm{ng} / \mathrm{mL}$ & 198 & & & \\
\hline PIVKA-II & & 0.010 & 0.425 & $0.217-0.829$ \\
\hline$\leq 5,080 \mathrm{mAU} / \mathrm{mL}$ & 412 & & & \\
\hline$>5,080 \mathrm{mAU} / \mathrm{mL}$ & 206 & & & \\
\hline \multicolumn{5}{|l|}{ Multivariate analysis } \\
\hline $\begin{array}{l}\text { Initial treatment } \\
\text { Sorafenib }\end{array}$ & & 0.005 & & \\
\hline LFP & & & 0.249 & $0.095-0.665$ \\
\hline $\begin{array}{l}\text { Performance status } \\
\quad 0\end{array}$ & & 0.017 & & \\
\hline 1 & & & 2.660 & $1.188-5.957$ \\
\hline Sex & & 0.356 & & \\
\hline Male & & & & \\
\hline Female & & & 0.498 & $0.113-2.187$ \\
\hline
\end{tabular}




\section{Liver Cancer}

Table 2 (continued)

Table 3. Grade 3 or higher adverse events and discontinuation due to adverse events

\begin{tabular}{l|l}
\hline \multicolumn{2}{l|}{ Liver Cancer 2017;6:275-286 } \\
\hline DOI: 10.1159/000473887 & $\begin{array}{l}\text { C 2017 S. Karger AG, Basel } \\
\text { www.karger.com/lic }\end{array}$ \\
\hline
\end{tabular}

Moriguchi et al.: Sorafenib versus Hepatic Arterial Infusion Chemotherapy as Initial Treatment for Hepatocellular Carcinoma with Advanced Portal Vein Tumor Thrombosis

\begin{tabular}{|c|c|c|c|c|}
\hline & MST & $p$ value & HR & $95 \%$ CI \\
\hline $\begin{array}{l}\text { Child-Pugh score } \\
\quad 5\end{array}$ & & $<0.001$ & & \\
\hline 6 & & & 4.204 & $1.903-9.288$ \\
\hline $\begin{array}{l}\text { Extrahepatic spread } \\
\text { Negative }\end{array}$ & & 0.059 & & \\
\hline Positive & & & 2.392 & $0.969-5.906$ \\
\hline $\begin{array}{c}\text { Main tumor size } \\
\leq 66.5 \mathrm{~mm}\end{array}$ & & 0.537 & & \\
\hline$>66.5 \mathrm{~mm}$ & & & 1.342 & $0.528-3.412$ \\
\hline $\begin{array}{l}\text { Tumor burden } \\
\leq 50 \%\end{array}$ & & 0.046 & & \\
\hline$>50 \%$ & & & 2.530 & $1.015-6.309$ \\
\hline $\begin{array}{l}\text { PIVKA-II } \\
\quad \leq 5,080 \mathrm{mAU} / \mathrm{mL}\end{array}$ & & 0.232 & & \\
\hline$>5,080 \mathrm{mAU} / \mathrm{mL}$ & & & 0.568 & $0.225-1.435$ \\
\hline
\end{tabular}

CI, confidence interval; HR, hazard ratio; LFP, low-dose 5-fluorouracil and cisplatin; MST, median survival time; PIVKA-II, protein induced by vitamin $\mathrm{K}$ absence or antagonist II; TACE, transarterial chemoembolization; $\mathrm{Vp}$, tumor thrombus involving the portal vein.

\begin{tabular}{lll}
\hline & LFP & Sorafenib \\
\hline Neutrophil count decreased & 4 & \\
Platelet count decreased & 8 & 1 \\
Aspartate aminotransferase increased & $1(3)$ & 2 \\
Alanine aminotransferase increased & $1(3)$ & 1 \\
Creatinine increased & 1 & \\
Amylase increased & & 1 \\
Hypophosphatemia & & 2 \\
Hyponatremia & & 2 \\
Diarrhea & 1 & 1 \\
Hepatic encephalopathy & & 1 \\
General malaise & 2 & 3 \\
Appetite loss & & 1 \\
Erythema exsudativum multiforme & 1 & \\
Hepatic artery occlusion & 1 & 7 \\
Cerebellar infarction & 3 & \\
Discontinuation due to adverse events & & \\
\hline
\end{tabular}

The numerical data represent the number of cases. Figures in parentheses represent the number of cases showing a transient increase after the placement of a reservoir catheter. There was a significant difference in the rate of discontinuation due to adverse events between the LFP group and the sorafenib group. LFP, low-dose 5-fluorouracil and cisplatin. 


\section{Liver Cancer}

Table 4. Child-Pugh score at the time of discontinuation due to tumor progression or at the time of discontinuation due to adverse events (a) and secondary treatments (b)

\begin{tabular}{l|l}
\hline Liver Cancer 2017;6:275-286 \\
\hline DOI: 10.1159/000473887 & $\begin{array}{l}\text { C 2017 S. Karger AG, Basel } \\
\text { www.karger.com/lic }\end{array}$ \\
\hline
\end{tabular}

Moriguchi et al.: Sorafenib versus Hepatic Arterial Infusion Chemotherapy as Initial Treatment for Hepatocellular Carcinoma with Advanced Portal Vein Tumor Thrombosis

\begin{tabular}{llll}
\hline a & C-P A & C-P 7 & C-P 8 \\
\hline Sorafenib $(n=14)$ & 2 & 7 & 5 \\
LFP $(n=27)$ & 19 & 5 & 3 \\
\hline b & & Sorafenib & LFP \\
& $(n=14)$ & $(n=27)$ \\
\hline Best supportive care & 11 & 16 \\
Sorafenib (continued) & $(2)$ & 6 \\
HAIC & 1 & 2 \\
Cisplatin & 1 & \\
5-FU continuous & & \\
Interferon + 5-FU & & 1 \\
TACE & & 1 \\
Resection of LN metastasis & & 2 \\
\hline
\end{tabular}

The numerical data represent the number of cases. Figures in parentheses represent the total number of cases of discontinuation due to adverse events and tumor progression. 5-FU, 5-fluorouracil; C-P, ChildPugh; HAIC, hepatic arterial infusion chemotherapy; LFP, low-dose 5-fluorouracil and cisplatin; LN, lymph node; TACE, transarterial chemoembolization. ${ }^{1}$ Continuous hepatic arterial infusion of 5 -fluorouracil using a reservoir catheter system.

be first administered for HCC with $\mathrm{Vp} 3 / \mathrm{Vp} 4$ because a better response can be expected. In the future, it would be important to determine, at an early time, whether to change treatments and introduce sorafenib as a secondary treatment for all cases in whom no response has been obtained. On the other hand, when sorafenib was administered as initial treatment, secondline treatment could not be administered in many cases because of a decline in hepatic function or PS. Grothey and Sargent [16] defended that making all active agents available to patients with advanced colorectal cancer was important to improve OS. This might be equally true for HCC with Vp3/Vp4. In other words, not only sorafenib, but also LFP should be provided to HCC patients with Vp3 as per consensus-based guidelines. In contrast, for Vp4, consensus-based guidelines in Japan recommend HAIC [8], while the Barcelona Clinic Liver Cancer (BCLC) guidelines recommend sorafenib [13]. However, neither of the 2 treatments alone provides satisfactory outcomes. Therefore, it would be desirable to provide both options. However, the outcome of this study suggests that LFP should be recommended as initial treatment for HCC with Vp3/Vp4.

In multivariate analysis, initial treatment was extracted as a significant factor contributing to OS. However, 6 patients received sorafenib as secondary treatment after LFP. For this reason, we compared the OS of LFP-treated patients who did not receive sorafenib as secondline treatment to that of patients treated with sorafenib alone. As a result, the former group had a significantly more favorable OS than the latter (MST, 309 vs. 120 days, respectively; $p=0.018$ ). Therefore, LFP seems to provide a survival benefit for HCC with Vp3/Vp4. Furthermore, EHS did not remain as a significant factor contributing to OS, and in an investigation according to presence or absence of EHS, with EHS-, the LFP group had a significantly more favorable prognosis as compared with the sorafenib group. Therefore, when considering whether to administer sorafenib or LFP as a treatment method for advanced HCC with Vp3/Vp4, at the very least if the case is EHS-, it is thought valid to select LFP. 
In subanalysis, in which the Vp3 and Vp4 groups were analyzed separately, there was no significant difference in OS between the LFP and the sorafenib group. However, this is thought to be due to the small number of cases. With regard to $\mathrm{Vp} 4$, the sample size was small in this study, but the $p$ value was 0.057 . Therefore, a potential benefit of LFP for HCC with Vp4 as compared with sorafenib has been suggested.

The limitations of our study are the fact that it is a single-institution retrospective study with few cases, and also that the initial dose of sorafenib was not consistent. Nevertheless, with regard to sorafenib dose, there were a number of cases in whom dose reduction or discontinuation could not be avoided due to adverse events. Therefore, it is thought that even if all cases had begun with the full dose, the same results would have likely been obtained.

In conclusion, given that (1) overall outcomes were favorable, (2) analysis of each of the Vp3 and Vp4 cases showed that LFP treatment had the same or better outcomes compared to those of sorafenib treatment, and (3) adverse events were manageable and the introduction of sorafenib was possible as a secondary treatment in most cases, it was thought that it is a valid option to select LFP as the initial treatment for Child-Pugh A advanced HCC with Vp3/ Vp4.

\section{Disclosure Statement}

Y. Itoh received a research grant from Esai Co., Ltd. and Bristol Myers Inc. The other authors declare no conflicts of interest.

\section{References}

1 Wilhelm SM, Carter C, Tang L, Wilkie D, McNabola A, Rong H, Chen C, Zhang X, Vincent P, McHugh M, Cao Y, Shujath J, Gawlak S, Eveleigh D, Rowley B, Liu L, Adnane L, Lynch M, Auclair D, Taylor I, Gedrich R, Voznesensky A, Riedl B, Post LE, Bollag G, Trail PA: BAY 43-9006 exhibits broad spectrum oral antitumor activity and targets the RAF/MEK/ERK pathway and receptor tyrosine kinases involved in tumor progression and angiogenesis. Cancer Res 2004;64:7099-7109.

2 Llovet JM, Ricci S, Mazzaferro V, Hilgard P, Gane E, Blanc JF, de Oliveira AC, Santoro A, Raoul JL, Forner A, Schwartz M, Porta C, Zeuzem S, Bolondi L, Greten TF, Galle PR, Seitz JF, Borbath I, Häussinger D, Giannaris T, Shan M, Moscovici M, Voliotis D, Bruix J; SHARP Investigators Study Group: Sorafenib in advanced hepatocellular carcinoma. N Engl J Med 2008;359:378-390.

3 Cheng AL, Kang YK, Chen Z, Tsao CJ, Qin S, Kim JS, Luo R, Feng J, Ye S, Yang TS, Xu J, Sun Y, Liang H, Liu J, Wang J, Tak WY, Pan H, Burock K, Zou J, Voliotis D, Guan Z: Efficacy and safety of sorafenib in patients in the AsiaPacific region with advanced hepatocellular carcinoma: a phase III randomised, double-blind, placebocontrolled trial. Lancet Oncol 2009;10:25-34.

4 Bruix J, Raoul JL, Sherman M, Mazzaferro V, Bolondi L, Craxi A, Galle PR, Santoro A, Beaugrand M, Sangiovanni A, Porta C, Gerken G, Marrero JA, Nadel A, Shan M, Moscovici M, Voliotis D, Llovet JM: Efficacy and safety of sorafenib in patients with advanced hepatocellular carcinoma: subanalyses of a phase III trial. J Hepatol 2012; 57:821-829.

5 Ando E, Tanaka M, Yamashita F, Kuromatsu R, Yutani S, Fukumori K, Sumie S, Yano Y, Okuda K, Sata M: Hepatic arterial infusion chemotherapy for advanced hepatocellular carcinoma with portal vein tumor thrombosis: analysis of 48 cases. Cancer 2002;95:588-595.

6 Lai YC, Shih CY, Jeng CM, Yang SS, Hu JT, Sung YC, Liu HT, Hou SM, Wu CH, Chen TK: Hepatic arterial infusion chemotherapy for hepatocellular carcinoma with portal vein tumor thrombosis. World J Gastroenterol 2003; 9:2666-2670.

7 Lin CC, Hung CF, Chen WT, Lin SM: Hepatic arterial infusion chemotherapy for advanced hepatocellular carcinoma with portal vein thrombosis: impact of early response to 4 weeks of treatment. Liver Cancer 2015; $4: 228-240$.

8 Kudo M, Matsui O, Izumi N, Iijima H, Kadoya M, Imai Y, Okusaka T, Miyayama S, Tsuchiya K, Ueshima K, Hiraoka A, Ikeda M, Ogasawara S, Yamashita T, Minami T, Yamakado K; Liver Cancer Study Group of Japan: JSH consensus-based clinical practice guidelines for the management of hepatocellular carcinoma: 2014 update by the Liver Cancer Study Group of Japan. Liver Cancer 2014;3:458-468.

9 Llovet JM, Bustamante J, Castells A, Vilana R, Ayuso Mdel C, Sala M, Brú C, Rodés J, Bruix J: Natural history of untreated nonsurgical hepatocellular carcinoma: rationale for the design and evaluation of therapeutic trials. Hepatology 1999;29:62-67. 
10 Villa E, Moles A, Ferretti I, Buttafoco P, Grottola A, Del Buono M, De Santis M, Manenti F: Natural history of inoperable hepatocellular carcinoma: estrogen receptors' status in the tumor is the strongest prognostic factor for survival. Hepatology 2000;32:233-238.

11 Song DS, Song MJ, Bae SH, Chung WJ, Jang JY, Kim YS, Lee SH, Park JY, Yim HJ, Cho SB, Park SY, Yang JM: A comparative study between sorafenib and hepatic arterial infusion chemotherapy for advanced hepatocellular carcinoma with portal vein tumor thrombosis. J Gastroenterol 2015;50:445-454.

12 Jeong SW, Jang JY, Shim KY, Lee SH, Kim SG, Cha SW, Kim YS, Cho YD, Kim HS, Kim BS, Kim KH, Kim JH: Practical effect of sorafenib monotherapy on advanced hepatocellular carcinoma and portal vein tumor thrombosis. Gut Liver 2013;7:696-703

13 Bruix J, Sherman M; American Association for the Study of Liver Diseases: Management of hepatocellular carcinoma: an update. Hepatology 2011;53:1020-1022.

14 Iavarone M, Cabibbo G, Piscaglia F, Zavaglia C, Grieco A, Villa E, Cammà C, Colombo M; SOFIA (SOraFenib Italian Assessment) Study Group: Field-practice study of sorafenib therapy for hepatocellular carcinoma: a prospective multicenter study in Italy. Hepatology 2011;54:2055-2063.

15 Ogasawara S, Chiba T, Ooka Y, Kanogawa N, Saito T, Motoyama T, Suzuki E, Tawada A, Kanai F, Yokosuka O: Sorafenib treatment in Child-Pugh A and B patients with advanced hepatocellular carcinoma: safety, efficacy and prognostic factors. Invest New Drugs 2015;33:729-739.

16 Grothey A, Sargent D: Overall survival of patients with advanced colorectal cancer correlates with availability of fluorouracil, irinotecan, and oxaliplatin regardless of whether doublet or single-agent therapy is used first line. J Clin Oncol 2005;23:9441-9442. 